\section{The role of}

computerised

tomography in

predicting visual

outcome in ocular trauma patients

Eye (2015) 29, 986; doi:10.1038/eye.2015.88

Correction to: Eye (2015) 29, 867-871; doi:10.1038/

eye.2015.39; published online 27 March 2015

Since the online publication of the above article, the authors have noted that an acknowledgement was missing from the article. Please find below the corrected Acknowledgements section with the missing text.

The study was carried out within the National Health Service (NHS). No extra financial support
R Chaudhary, M Upendran, N Campion, A Yeung, R Blanch, P Morgan-Warren, I Gibb, T Nelson and R Scott

was sought or needed to complete the study. We would like to acknowledge the National Institute for Health Research Surgical Reconstruction and Microbiology Research Centre, Queen Elizabeth Hospital Birmingham.

The authors would like to apologise for this error.

This error has now been rectified, and the corrected article appears in this issue. The html and online pdf versions have also been rectified, and now carry the corrected paper. 